\title{
Percepción de la Seguridad Alimentaria de los hogares del municipio San Francisco Libre, Managua 2014-2016
}

M.Sc. Carmen María Flores Machado

Doctorante en Desarrollo Rural Territorial Sustentable UNAN-Managua, FAREM-Matagalpa carmenflores280165@yahoo.com

Fecha de recepción: 12 de noviembre, 2019 / Fecha de aceptación: 22 de noviembre, 2019 https://doi.org/10.5377/torreon.v8i22.9024

Financiamiento de Fondos Proyectos de Investigación de la UNAN-Managua.

Palabras clave: territorio, seguridad alimentaria, ELCSA

\section{RESUMEN}

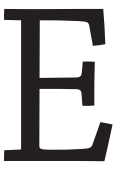

1 estudio sobre la percepción de la seguridad alimentaria es una contribución al análisis de la Seguridad Alimentaria y Nutricional desde el ámbito local para identificar a los grupos de riesgo y de mayor vulnerabilidad. La investigación fue descriptiva y observacional donde se examinaron las características socio demográficas de los jefes de hogar, el acceso y consumo de los alimentos en el hogar, y la suficiencia alimentaria de los hogares de las comunidades: Las Delicias, San Roque y La Huertas y del casco urbano de la cabecera departamental del municipio. Entre los principales resultados se destacan que el $78 \%$ de los jefes del hogar son hombres, de estos, agricultores el 52\% y su núcleo familiar integrado por 4,2 miembros. El 86\% de los hogares sufren inseguridad alimentaria por la falta de acceso a los alimentos en cantidad y calidad adecuada para tener una vida sana en el periodo estudiado. Con mayor grado de severidad en los niveles (inseguros leve y moderado) con un tercio respectivamente de los hogares urbanos y rurales. Lo que evidencia que los hogares han 
experimentado incertidumbre y preocupación por la falta de alimentos inclusive hambre por la falta de dinero y recursos para acceder a los alimentos en cantidad suficiente y calidad adecuada para gozar de una alimentación saludable. El nivel de suficiencia energético es crítico para el 40\% de los hogares y nivel de suficiencia alimentaria deficiente para el 20\% de los hogares rurales y urbanos del municipio.

\section{INTRODUCCIÓN}

Nicaragua a través del Instituto Nacional de Información de Desarrollo ha tenido una costumbre de medir las necesidades alimentarias de la población desde las encuestas de ingresos/ gastos para determinar el consumo de alimentos. Se manejan desde un marco de gastos en porcentajes por parte de los hogares. Si bien tienen toda condición técnica para una credibilidad, también no deja de ser verdad que los datos que se producen están basados en lo económico. Las encuestas que se realizan hasta el momento no consideran la seguridad alimentaria en el hogar (SAH), y deberían ser el mecanismo para cuantificar las necesidades y consumo real de alimentos desde una visión de acceso seguro a los mismos en todo momento en cantidad y calidad necesarias para que los miembros del hogar puedan desarrollar una vida sana, productiva y activa. (Instituto Nacional de Estadisticas y Censo, 2004).

El concepto de Seguridad Alimentaria Familiar surge a finales de los años ochenta y se ha mantenido dominante desde entonces en los debates teóricos, al ser progresivamente asumido por círculos académicos y por diferentes organismos internacionales (FAO, Banco Mundial, agencias bilaterales de ayuda). Este enfoque implica una doble reorientación. Toma como escala a la familia y se centra en el acceso a los alimentos determinado por el grado de vulnerabilidad socioeconómica (Carrazón, 2012).

La medición para la experiencia de los hogares frente a la inseguridad alimentaria se ha extendido en todos los continentes particularmente en América Latina y el Caribe a partir de las experiencias de los países que han contribuidos en el surgimiento de la Escala Latinoamericana y caribeña (ELCSA) para una mejor comprensión de la distribución, causas y consecuencias de la inseguridad alimentaria del hogar. La ELCSA es un instrumento de bajo costo y rápida aplicación que ha mostrado tener una validez y confiabilidad; aplicada con éxitos en los ámbitos nacional y local en encuestas gubernamentales, estudios académicos y/o sondeos de opinión pública (FAO, 2012).

En Centroamérica el $14.2 \%$ de la población están subnutridas, al compararse con América Latina y el Caribe tiene un $5 \%$ por encima de la media de ALC. La región es una de las zonas más vulnerables en término de subnutrición dentro del continente americano. Para el caso de Nicaragua, la subnutrición representa el 19 \% de su población y la desnutrición crónica en menores de cinco años el 22 \% (PRESANCA II y FAO, 2011). 
Es así que el estudio sobre la valoración de la percepción de la seguridad alimentaria en los hogares del municipio San Francisco Libre aporta evidencias para el diseño de intervenciones integrales que contribuyan a mejorar esta condición de inseguridad alimentaria de los hogares y contribuir a la prevención de la vulnerabilidad nutricional por parte de la municipalidad.

\section{MATERIALES Y METODOS}

El estudio según el nivel inicial de profundidad es descriptivo y según el método es observacional (Piura, 2006). De acuerdo al tiempo de ocurrencia de los hechos y registro de la información es retrospectivo y según el periodo y secuencia del estudio es transversal (Pineda, 2008). La muestra fue de 119 hogares.

El Municipio de San Francisco Libre pertenece al departamento de Managua, tiene una extensión territorial de $756 \mathrm{~km}^{2}$ con una posición geográfica de latitud norte $12^{\circ} 30^{\prime}$ y longitud $85^{\circ} 18$ oeste a 40 ( $\mathrm{msnm}$ ) de altitud sobre el nivel del mar. El municipio ocupa el tercer lugar en extensión territorial de los nueve municipios que componen el departamento de Managua. En particular, la cabecera municipal de San Francisco Libre ubicado en la orilla del lago de Managua con la comarca de Laurel Galán, ubicada en la zona norte, la comarca Telpochapa, en la zona centro oriental, y la comarca de San Roque en la zona sur del municipio.

El municipio se encuentra en la zona del trópico seco de Nicaragua ubicada en la región baja del pacifico con un promedio de precipitaciones anuales que oscilan entre los 1,000-1500 $(\mathrm{mm})$ milímetros anuales y presenta un patrón irregular caracterizado por temporada de exceso o de escasez de agua. Desde el punto hidrológico, tienen tres cuencas que son parte de la cuenca de San Juan (Lago de Managua). Los suelos son escarpados donde se localizan numerosos cerros mayormente de tipo franco-arcillosos y arcillosos (suelos pesados que presentan baja permeabilidad al agua y elevada retención de agua y nutrientes) con erosión moderada y mayormente con cobertura agropecuaria.

El municipio de San Francisco Libre se encuentra ubicado en el círculo más inmediato que rodea Managua, con una población de 10,949 habitantes, con predominio de mujeres. Existe pobreza extrema en el $34 \%$ de los hogares, con marcada incidencia entre Barrios y Comarcas. El 76 \% de los hogares son del área rural (Instituto Nacional de Información para el Desarrollo, 2008).

El universo estuvo constituido por 1,968 hogares de las tres comarcas y el casco urbano de la cabecera municipal. El área urbana está dividido en 3 barrios y el área rural con 3 comarcas: Las Delicias, San Roque y La Huertas. Para determinar el tamaño de la muestra se aplicó la fórmula de Mounch Galindo para poblaciones finitas, y se obtuvo una muestra de 119 hogares 
con un 95\% del nivel de confianza y un 5\% de error muestral. El procesamiento de la información se hizo a través del programa estadístico SPSS Version25 para Windows.

La escala ELCSA es un instrumento de medición indirecta de la seguridad alimentaria en el hogar, de bajo costo, que ha demostrado validez y confiabilidad en diversos canales de aplicación. En el nivel local hasta el nivel nacional ha tenido mucho éxito en comprensión de la distribución, causas y consecuencia de la inseguridad alimentaria en la región. Mide los grados de severidad de la inseguridad alimentaria (leve, moderada, severa) y es un instrumento confiable que permite optimizar y focalizar alertas tempranas en políticas de prevención (FAO, 2012).

El marco conceptual de la Inseguridad Alimentaria en el hogar contiene cuatro componentes: inicialmente existe ansiedad y preocupación por los suministros de alimentos; a continuación, se hace ajustes en el presupuesto del hogar, afectando la calidad de la dieta; tercero, los adultos limitan la calidad y la cantidad de los alimentos que se consumen; y finalmente se afectan la calidad y cantidad de los alimentos consumidos por los niños. Para determinar la inseguridad en el hogar, la ELCSA está constituida por 15 preguntas (P), divididas en dos secciones: una primera con 8 preguntas (P1-P8) son aplicables para los hogares con adultos, y una segunda sección (P9- P15) con preguntas referidas a condiciones que afectan a los menores de 18 años en el hogar. Cada pregunta está dirigida a indagar sobre una situación diferente, por lo que se trata de preguntas excluyentes, y cada una de ellas pretende captar distintos asuntos relacionados con el constructo teórico que respalda a la ELCSA, (FAO, 2012).

Consentimiento Informado: Los jefes de los hogares participantes firmaron el consentimiento informado, el cual incluyó los principios éticos para las investigaciones médicas en seres humanos de la declaración de Helsinki.

\section{RESULTADOS Y DISCUSION: TABLAS Y GRAFICOS}

En cuanto a las características socio demográficas de los jefes de hogares, el $42 \%$ están casados y el $31 \%$ viven en unión de hecho con su pareja; el 22\% no completaron estudios de educación primaria y el $28,2 \%$ son analfabetas. La composición familiar integrada por 4.2 personas y el 66 \% son mayores de 18 años y 34\% menores de 18 años. Es importante destacar que el 52\% son agricultores dedicados a la producción de granos básicos y crianzas de animales.

La medición de la seguridad alimentaria en los hogares mediante la ELCSA evidencia inseguridad alimentaria en el 86 \% de los hogares urbanos y rurales del municipio San Francisco Libre. Los hogares presentan distintos grados de severidad de Inseguridad Alimentaria (leve, moderada y crítica) y solo $14 \%$ tienen seguridad alimentaria en los hogares de las comunidades: Las Huertas, Las Lomas y Las Delicias y casco urbano. Al desagregarlos por los niveles de severidad: el $35 \%$ son inseguro leve, el $30 \%$ es inseguro moderada y el $21 \%$ es inseguro severo 
de los 119 hogares. Las razones de padecer inseguridad alimentaria son faltas de dinero y recursos para acceder a los alimentos que necesitan para garantizar una alimentación saludable que permitan obtener el máximo provecho de sus potenciales y capacidades para una vida digna. La figura 1 presenta la prevalencia de Inseguridad Alimentaria de los hogares del municipio por grado de severidad.

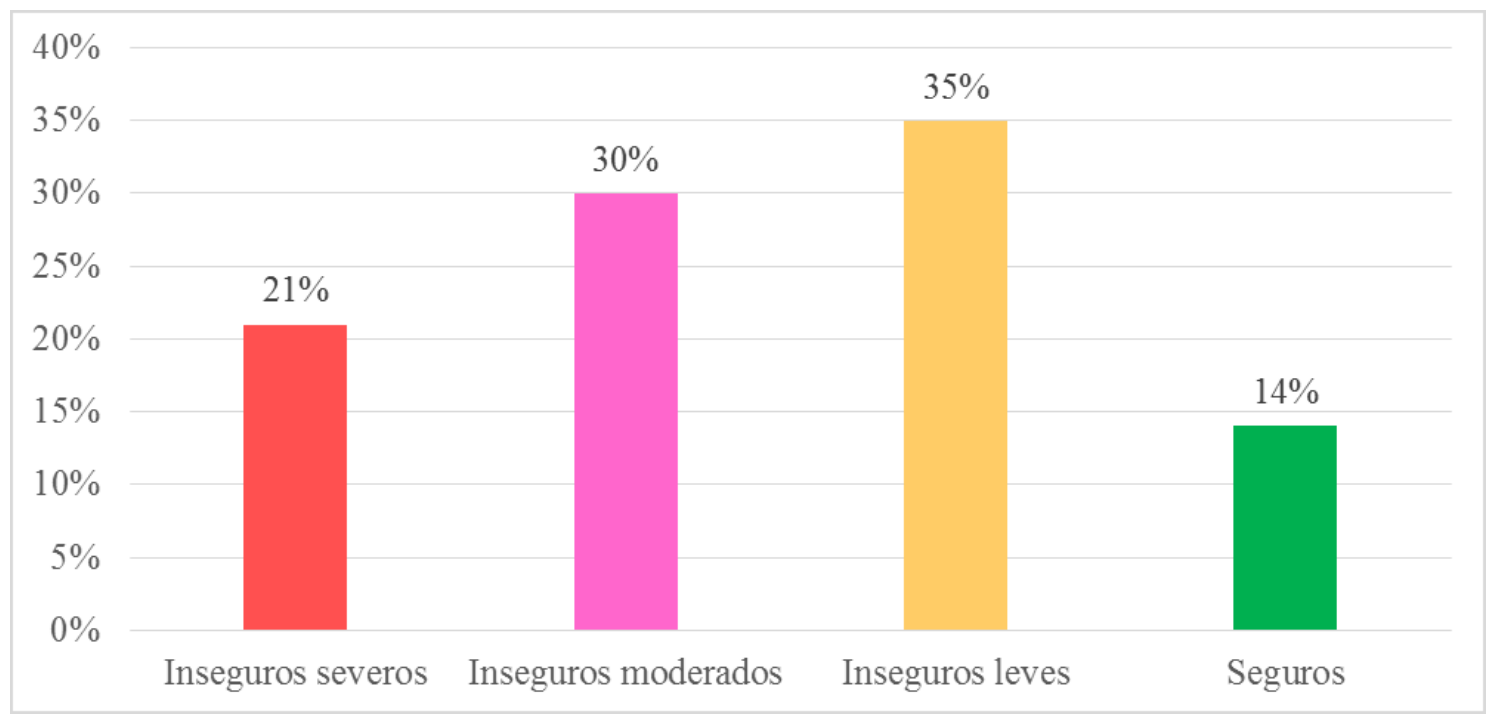

Figura 1. Prevalencia de la inseguridad alimentaria en hogares San Francisco Libre, Managua, 2014- 2015. Fuente: Encuesta

La inseguridad alimentaria por grupo de edades entre mayores de 18 años y menores de 18 años. Estos últimos con una marcada falta de acceso a los alimentos principalmente de las categorías inseguro leve con $40 \%$, inseguro moderado $26 \%$ e inseguro severo $20 \%$ La reducción de consumo de alimentos es muy marcada para los menores de 18 años donde los niños y jóvenes están más expuestos a la reducción de alimentos y hambre afectando la calidad de la alimentación y por consiguiente afectando su estado de nutrición y salud.

En cambio, en los hogares con solo adultos se acentúa el nivel de inseguro moderado y severo con $41 \%$ y $22 \%$ respectivamente, se muestra que los adultos han estado sometidos a mayor periodo de limitaciones en la ingesta de alimentos tanto en la cantidad y calidad de los alimentos y dan prioridad a los menores de 18 años. Toda esta condición es resultado de una disponibilidad insuficiente de alimentos, baja capacidad adquisitiva para comprar los alimentos y por consiguiente un limitado consumo familiar de alimentos suficientes, nutritivos e inocuos para tener una vida saludable. La tabla 1 muestra los tipos de hogares según categorías de la inseguridad alimentaria. 
Tabla 1. Tipos de hogares según categorías de inseguridad alimentaria San Francisco Libre 2014- 2015 Fuente Encuesta

\begin{tabular}{|l|c|c|c|c|c|c|c|c|c|c|}
\hline & \multicolumn{2}{|c|}{ Seguros } & \multicolumn{2}{c|}{$\begin{array}{c}\text { Inseguros } \\
\text { Leves }\end{array}$} & \multicolumn{2}{c|}{$\begin{array}{c}\text { Inseguros } \\
\text { Moderados }\end{array}$} & \multicolumn{2}{c|}{$\begin{array}{c}\text { Inseguros } \\
\text { Severos }\end{array}$} & \multicolumn{2}{c|}{} \\
\cline { 2 - 11 } & Casos & $\%$ & Casos & $\%$ & Casos & $\%$ & Casos & $\%$ & Casos & $\%$ \\
\hline $\begin{array}{l}\text { Hogares con } \\
\text { menores de } \\
18 \text { años }\end{array}$ & 12 & $14 \%$ & 35 & $40 \%$ & 23 & $26 \%$ & 17 & $20 \%$ & 87 & $100 \%$ \\
\hline $\begin{array}{l}\text { Hogares } \\
\text { adultos }\end{array}$ & 4 & $13 \%$ & 7 & $22 \%$ & 13 & $41 \%$ & 8 & $25 \%$ & 32 & $100 \%$ \\
\hline Total & 16 & $13 \%$ & 42 & $35 \%$ & 36 & $30 \%$ & 25 & $21 \%$ & 119 & $100 \%$ \\
\hline
\end{tabular}

Los hogares de San Francisco Libre enfrentan situaciones de carencias alimentarias. El $78 \%$ de los hogares con adultos presentan preocupación por los alimentos antes que se acaben, el $42 \%$ no tenían alimentos para comer al momento del periodo estudiado, el $63 \%$ dijeron no tener una alimentación balanceada, el 66 \% dijeron tener una alimentación basada en poca variedad de alimentos, el 30 \% no realizó algún tiempo de comida por la falta de alimentos, el $44 \%$ comieron menos de lo que correspondería comer por la falta de dinero o recursos, el 36 \% comió menos de los que debería comer y el 24 \% solo comieron una vez al día por la falta de recursos.

El $40 \%$ de los hogares con menores de 18 años no tuvieron una alimentación saludable, el $44 \%$ no tuvieron una alimentación variada, el $16 \%$ por la falta de alimentos omitieron algún tiempo de comida, el $21 \%$ de los hogares comieron menos de lo recomendado, el $23 \%$ de ellos disminuyeron la cantidad de alimentos servido en alguna de las comidas por la falta de dinero o recurso, el $17 \%$ de los hogares sintieron hambre y el $15 \%$ solo comieron una vez al día o dejaron de comer durante todo un día. Siendo más acentuada la vulnerabilidad alimentaria en los hogares solo con adultos.

En todos los hogares se experimenta la preocupación por los alimentos, y esta continúa con más fuerza cuando el acceso a los alimentos disminuye por la falta de dinero o recursos para comprarlos llevándolo a comprar alimentos rendidores sin tomar en cuenta el criterio nutricional por la falta de recursos. Dejando aún lado gozar de una alimentación saludable hasta llegar a disminuir la cantidad de alimentos que preparan el consumo familiar por omisión de algún tiempo de comida o bien reducción de la porción de alimento conllevando al detrimento de la calidad nutritiva de la dieta y a padecer hambre en algún momento del periodo estudiado. 


\section{PERCEPCIÓN DE LA SEGURIDAD ALIMENTARIA (ELCSA)}

Tabla 2. Tipos de hogares según categorías de inseguridad alimentaria San Francisco Libre 2014- 2015. Fuente: Encuesta

\begin{tabular}{|l|l|l|l|l|l|}
\hline & \multicolumn{3}{|c|}{ Hogares } & \multirow{2}{*}{ Total } \\
\cline { 2 - 5 } & $\begin{array}{c}\text { Hogares con } \\
\text { menores de } \\
\text { 18 años }\end{array}$ & \multicolumn{2}{|c|}{$\begin{array}{l}\text { Hogares } \\
\text { adultos }\end{array}$} & \\
\cline { 2 - 5 } & Casos & $\%$ & Casos & $\%$ & \\
\hline $\begin{array}{l}\text { 1. En los últimos 6 meses, por falta de dinero u } \\
\text { otros recursos, alguna vez, usted se preocupó por- } \\
\text { que los alimentos se acabaran en su hogar }\end{array}$ & 67 & $89 \%$ & 26 & $93 \%$ & 93 \\
\hline $\begin{array}{l}\text { 2.En los últimos 6 meses, por falta de dinero u } \\
\text { otros recursos, alguna vez, en su hogar se queda- } \\
\text { ron sin alimentos }\end{array}$ & 33 & $44 \%$ & 17 & $61 \%$ & 50 \\
\hline $\begin{array}{l}\text { 3.En los últimos 6 meses, por falta de dinero u } \\
\text { otros recursos, alguna vez, en su hogar no tuvie- } \\
\text { ron una alimentación saludable y nutritiva }\end{array}$ & 52 & $69 \%$ & 23 & $82 \%$ & 75 \\
\hline $\begin{array}{l}\text { 4.En los últimos 6 meses, por falta de dinero u } \\
\text { otros recursos, alguna vez, usted o algún adulto } \\
\text { de su hogar tuvo una alimentación con poca varie- } \\
\text { dad de alimentos }\end{array}$ & 55 & $73 \%$ & 24 & $86 \%$ & 79 \\
\hline $\begin{array}{l}\text { 5.En los últimos 6 meses, por falta de dinero u } \\
\text { otros recursos, alguna vez, usted o algún adulto } \\
\text { de su hogar dejó de desayunar, almorzar o cenar }\end{array}$ & 23 & $31 \%$ & 13 & $46 \%$ & 36 \\
\hline $\begin{array}{l}\text { 6.En los últimos 6 meses, por falta de dinero u } \\
\text { otros recursos, alguna vez, usted o algún adulto } \\
\text { de su hogar comió menos de lo que debía comer }\end{array}$ & 37 & $49 \%$ & 16 & $57 \%$ & 53 \\
\hline $\begin{array}{l}\text { 7.En los últimos 6 meses, por falta de dinero u } \\
\text { otros recursos, alguna vez, usted o algún adulto } \\
\text { de su hogar sintió hambre pero no comió }\end{array}$ & 28 & $37 \%$ & 15 & $54 \%$ & 43 \\
\hline $\begin{array}{l}\text { 8.En los últimos 6 meses, por falta de dinero u } \\
\text { otros recursos, alguna vez, usted o algún adulto } \\
\text { de su hogar solo comió una vez al día o dejó de } \\
\text { comer durante todo un día }\end{array}$ & 20 & $27 \%$ & 9 & $32 \%$ & 29 \\
\hline
\end{tabular}




\begin{tabular}{|c|c|c|c|c|c|}
\hline & \multicolumn{4}{|c|}{ Hogares } & \multirow[t]{3}{*}{ Total } \\
\hline & \multicolumn{2}{|c|}{$\begin{array}{l}\text { Hogares con } \\
\text { menores de } \\
18 \text { años }\end{array}$} & \multicolumn{2}{|c|}{$\begin{array}{l}\text { Hogares } \\
\text { adultos }\end{array}$} & \\
\hline & Casos & $\%$ & Casos & $\%$ & \\
\hline $\begin{array}{l}\text { 9.En los últimos } 6 \text { meses, por falta de dinero u } \\
\text { otros recursos, alguna vez, algún menor de } 18 \\
\text { años de su hogar dejó de tener una alimentación } \\
\text { saludable y nutritiva }\end{array}$ & 45 & $60 \%$ & 3 & $11 \%$ & 48 \\
\hline $\begin{array}{l}\text { 10.En los últimos } 6 \text { meses, por falta de dinero u } \\
\text { otros recursos, alguna vez, algún menor de } 18 \\
\text { años de su hogar tuvo una alimentación con poca } \\
\text { variedad de alimentos }\end{array}$ & 50 & $67 \%$ & 3 & $11 \%$ & 53 \\
\hline $\begin{array}{l}\text { 11.En los últimos } 6 \text { meses, por falta de dinero u } \\
\text { otros recursos, alguna vez, algún menor de } 18 \\
\text { años de su hogar dejó de desayunar, almorzar o } \\
\text { cenar }\end{array}$ & 19 & $25 \%$ & 1 & $4 \%$ & 20 \\
\hline $\begin{array}{l}\text { 12.En los últimos } 6 \text { meses, por falta de dinero u } \\
\text { otros recursos, alguna vez, algún menor de } 18 \\
\text { años de su hogar comió menos de lo que debía } \\
\text { comer }\end{array}$ & 25 & $33 \%$ & 1 & $4 \%$ & 26 \\
\hline $\begin{array}{l}\text { 13.En los últimos } 6 \text { meses, por falta de dinero u } \\
\text { otros recursos, alguna vez, Tuvieron que dismi- } \\
\text { nuir la cantidad servida en las comidas a algún } \\
\text { menor de } 18 \text { años de su hogar }\end{array}$ & 27 & $36 \%$ & 1 & $4 \%$ & 28 \\
\hline $\begin{array}{l}\text { 14.En los últimos } 6 \text { meses, por falta de dinero u } \\
\text { otros recursos, alguna vez, algún menor de } 18 \\
\text { años de su hogar sintió hambre pero no comió }\end{array}$ & 19 & $25 \%$ & 2 & $7 \%$ & 21 \\
\hline $\begin{array}{l}\text { 15.En los últimos } 6 \text { meses, por falta de dinero u } \\
\text { otros recursos, alguna vez, algún menor de } 18 \\
\text { años de su hogar solo comió una vez al día o dejó } \\
\text { de comer durante todo un día }\end{array}$ & 16 & $21 \%$ & 2 & $7 \%$ & 18 \\
\hline Total & 75 & & 28 & & 103 \\
\hline
\end{tabular}

El patrón de consumo de alimentos está integrado por 12 alimentos. Los alimentos más consumidos son: pan simple, café, avena, huevos, pollo, pan dulce, queso, gaseosas y pinolillos, banano, naranja y plátanos verdes por más del $50 \%$ de las familias. Estas lo consumen más 
de tres veces por semana durante el último año, en su mayoría son productos energéticos, misceláneos como las gaseosas fuentes de calorías vacías, carnes de pollo fuentes importantes de proteínas de origen animal en más $60 \%$, los productos lácteos y huevos también consumido por más del $66 \%$ de los hogares. Al igual que el grupo de las carnes de aves.

Los alimentos más consumidos son tres, el 73 \% de los hogares: pan, café y avena perteneciente a los grupos de cereales y misceláneos. El grupo de las frutas y vegetales lo consumen el $50 \%$ de los hogares. El patrón de consumo es altamente energético con poca variedad de alimentos y tiene una correspondencia con el patrón de consumo de alimentos para el área rural integrado por 15 productos (Ministerio de Agricultura, Ganaderia y Forestal MAGFOR, 2004). Con cierta diferencia en ciertos productos, por ejemplo, el pan simple y dulce ha ganado terreno en la preferencia por los hogares al igual el consumo de huevos y carnes de pollo en contraposición del consumo de tortillas de maíz, plátanos y arroz.

Tabla 3. Patrón de consumo de más del 50 \% de los hogares San Francisco Libre, Managua

\begin{tabular}{|l|c|c|}
\hline \multicolumn{1}{|c|}{ Alimentos consumidos por el 50\% de los hogares encuestados } \\
\hline \multicolumn{1}{|c|}{ Alimes } & No de hogares & $\%$ \\
\hline $\begin{array}{l}\text { Pan simple (francés, pan molde, bollón, } \\
\text { otros) }\end{array}$ & 87 & $73 \%$ \\
\hline Café o té & 87 & $73 \%$ \\
\hline $\begin{array}{l}\text { Cereal de desayuno (avena, semilla de jícaro, } \\
\text { cebada) }\end{array}$ & 86 & $72 \%$ \\
\hline Huevos de gallina u otras aves & 79 & $66 \%$ \\
\hline Carne de gallina o pollo (incluyendo vísceras) & 78 & $66 \%$ \\
\hline $\begin{array}{l}\text { Pan dulce (picos, semitas, tortas, bonete, } \\
\text { otros) }\end{array}$ & 77 & $65 \%$ \\
\hline $\begin{array}{l}\text { Queso (cualquier tipo Quesillo, cuajada, re- } \\
\text { quesón, crema otros) }\end{array}$ & 76 & $64 \%$ \\
\hline Gaseosas & 65 & $55 \%$ \\
\hline Pinolillo, pinol o pozol & 64 & $54 \%$ \\
\hline Banano & 58 & $50 \%$ \\
\hline Frutas cítricas ( naranja) & $58 \%$ \\
\hline $\begin{array}{l}\text { Plátano verde, plátano maduro y guineo } \\
\text { cuadrado }\end{array}$ & & $50 \%$ \\
\hline
\end{tabular}


El nivel de suficiencia alimentaria en los hogares de San Francisco Libre es del 38\% y entre los niveles deficientes el $22 \%$ y nivel crítico el $40 \%$ de los hogares, estas adecuaciones son consideradas niveles críticos de inseguridad alimentaria y posiblemente estén vinculados a niveles críticos de vulnerabilidad nutricional. En Nicaragua la adecuación energética se clasifica en cuatro niveles: nivel suficiente con una adecuación de 110 a 150 \%, nivel aceptable con una adecuación de 90 a 109 \%, el nivel deficiente con una adecuación de 70 a 89 \% y nivel crítico con una adecuación menor de 70 \% de la suficiencia energética proveniente de la dieta.

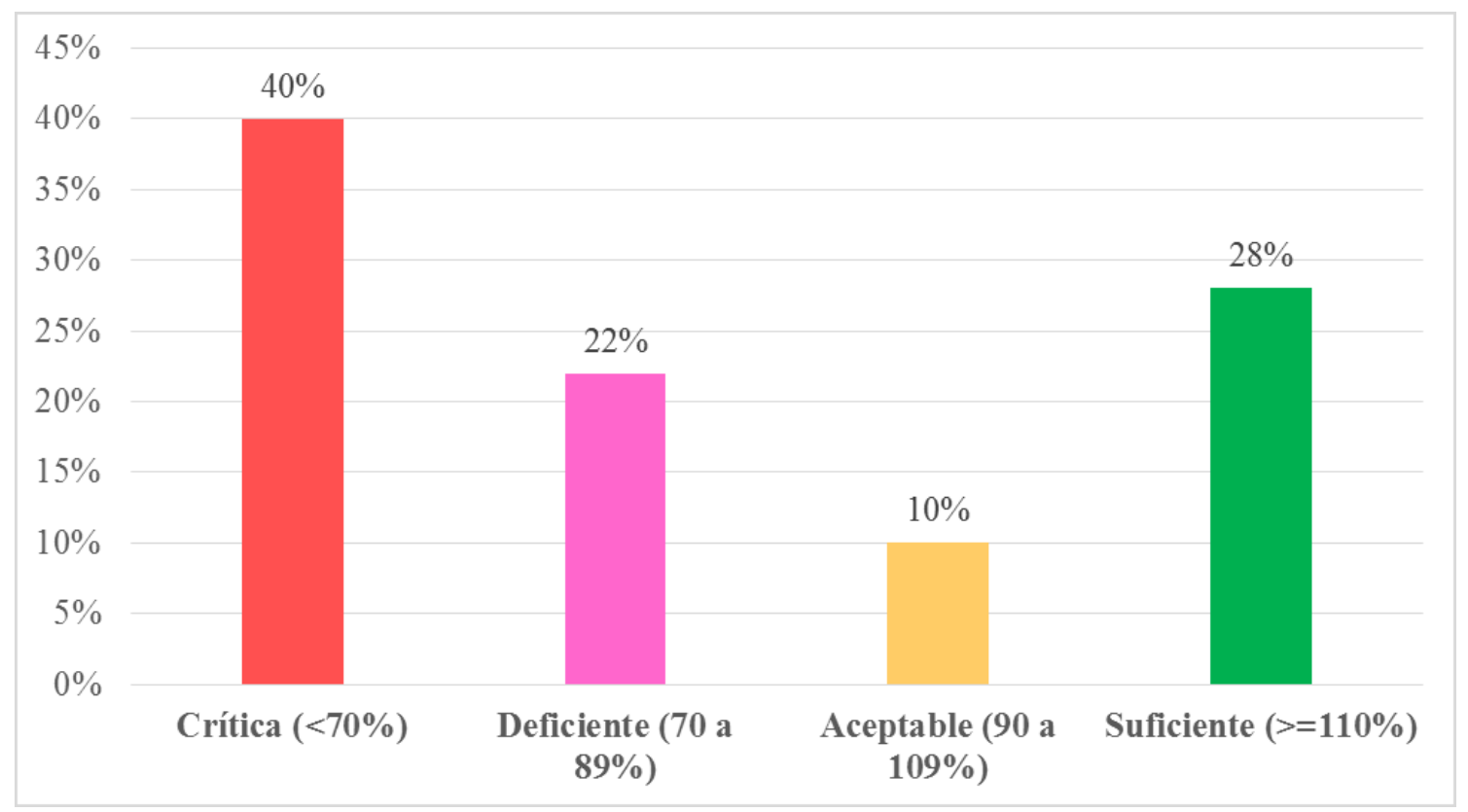

Figura 2. Niveles de Suficiencia Energética de los hogares San Francisco Libre, Managua 2014- 2015

\section{CONCLUSIONES}

Existen un predominio de sexo masculino en los jefes de hogar y el $55 \%$ tiene un nivel de escolaridad de primaria incompleta. La ocupación que predomina en primer lugar es agricultor con el 52 \%, seguido $13 \%$, luego obrero y comerciantes el 14 \%. La composición familiar integrada por 4.2 miembros.

El 86 \% de los hogares están en Inseguridad Alimentaria por la falta de dinero y recursos para acceder a los alimentos de forma suficiente y cantidad necesaria para tener una alimentación saludable.

Los hogares se encuentran en distintos grados de inseguridad alimentaria, siendo mayor los niveles de inseguros leve con el 35\%, inseguros moderado con el $30 \%$ e inseguros severo del $21 \%$ para los hogares con menores de 18 años y adultos.

La inseguridad alimentaria es más acentuada en los grados moderado y severo en los hogares adultos, conjeturando que los adultos dan prioridad a los menores de 18 años en no afectar la cantidad y calidad de alimentos a consumir por este grupo. 
El patrón de consumo de alimento es limitado y poco variado constituido por 12 alimentos: pan simple, café, avena, huevos, pollo, pan dulce, queso, gaseosas y pinolillos, banano, naranja y plátanos verdes. Con una contribución energética e calorías vacías significativa, modesto aporte proteínico y deficiente aporte de vitaminas y minerales.

El nivel de suficiencia energética de los alimentos consumidos por los hogares en el periodo de estudio fue del $38 \%$ clasificado como aceptable el resto de los hogares tienen nivel de suficiencia crítico con el $40 \%$ y suficiencia alimentaria deficiente el 22\% de los hogares.

\section{BIBLIOGRAFIA}

Alcaldia de Managua. (2005). Caracterización del distrito 3 Managua. Managua .

Carrazón, j. G. (2012). Seguridad Alimentaria para Todos. España: Visión Libros.

FAO. (2012). Escala Latinoamericana y Caribeña de Seguridad Alimentaria (ELCSA). Roma.

FAO. (2013). Desarrollo Territorial Participativo y Negociado. FAO.

INIDE. (2014). ENDESA.

INIDE. (s.f.) Alcaldia de Managua. Recuperado el 2016, de Caracteristicas Generales del municipio de Managua por distritos: http://www.managua.gob.ni/modulos/ documentos/caracterizacion.pdf

Instituto Nacional de Estadisticas y Censo. (2004). Analísis de la Pobreza y la Seguridad Alimentaria y Nutricional en Nicaragua. Nicaragua. Obtenido de https://www. sica.int/presanca1/informe_final/ P RE S A N C A \% 20 I N F O R M E \% 20 FINAL\%20MARZO2010.pdf
Instituto Nicaraguense de Información para el Desarrollo. (2008). San Franciscio Libre en Cifras. Nicaragua. Obtenido de https://www.inide.gob.ni/censos2005/ Cifras Mun / Managua / S a n \% 20 Francisco\%20Libre.pdf

Ministerio de Agricultura, Ganaderia y Forestal, MAGFOR. (2004). IV Encuesta de consumo de alimentos de Nicaragua. Nicaragua: Impresión Comercial La Prensa.

PRESANCA II y FAO. (2011). Centroamérica en Cifras. Guatemala. 